\title{
Circuits for Optical Based Line of Sight Voice Communication
}

\author{
Devasis Haldar ${ }^{*}$, Shagun Panwar, Vipul Kumar, Ayush Goswami, Sakshi Dhawan \\ Department of Electronics and Communication Engineering, SRM University NCR campus, India \\ ${ }^{*}$ Corresponding author, e-mail: devasishaldar62@gmail.com
}

\begin{abstract}
We present here line of sight communication between a person and his neighbour with the help of optical signal produced by a laser torch which act as a carrier. It is therefore a wireless communication and the transmission can go up to 500 meters. We used photodiode to receive the signal at the receiver. The transmitter circuit comprises condenser microphone transistor amplifier BC547 followed by an op-amp stage built around $\mu A 741$. When we give a voice signal from the mike, it converts the voice signal into the electrical signal. This electrical signal is fed to IC741 (op-amp) for amplification. The gain of the op-amp can be controlled with the help of 1-mega-ohm potentiometer. The AF output from IC is coupled to the base of a class $B$ amplifier which, in turn, modulates the signal. The transmitter uses $5 \mathrm{~V}$ power supply. However, the 3-volt laser torch (after removal of its battery) can be directly connected to the circuit-with the body of the torch connected to the class $B$. The photodiode converts the optical signal into electrical signal and again this signal is amplified using IC741 and a combination of class B push pull amplifiers. The receiver circuit uses an NPN photodiode as the light sensor that is followed by a two-stage transistor preamplifier and IC741 based audio Power amplifier. The receiver does not need any complicated alignment. Just keep the photodiode oriented towards the remote transmitter's laser point and adjust the volume control for a clear sound. The sensor must not directly face the sun.
\end{abstract}

Keywords: laser, NPN, diode

\section{Introductory on Laser}

Laser the Light Amplification by Stimulated Emission of Radiation, uses the process of stimulated emission is used for amplifying light waves. Other processes are absorption and spontaneous emission. The rate of absorption and emissions are related by means of Einstein's relations.

A laser diode, or LD, is an electrically pumped semiconductor laser in which the active laser medium is formed by a p-n junction of a semiconductor diode similar to that found in a light-emitting diode [1]. However the lasers are characterized by higher power and narrow line width. Laser action in semiconductors may be achieved by forming an optical cavity in the active region of a DH (Double Heterostructure). The difference with DH LED is that the end faces of the crystal forming the active region along the longitudinal direction are cleaved so that they can act as mirrors. In some cases, mirror coating is deposited on one side to make the reflectivity nearly unity. The electrons are injected from the $\mathrm{N}$-region into the conduction band of the $\mathrm{p}$ region and holes are injected from the P-region into the valence band of the p-region. As a result population inversion occurs corresponding to transition between those levels for which the photon energy is greater than the band gap energy of the active region, but is less than the energy difference between quasi Fermi levels. The refractive index of the active region is greater than that of the confining layer, and hence optical confinement is provided in the transverse direction, but optical feedback is provided in longitudinal direction. Thus laser action takes place along longitudinal direction.

High data rate, absence of recurring line costs, absence of shocks, short circuit, ground loops, non requirement of routers, narrow beam divergence, service free optical wireless etc. are advantages of laser transmission over electrical transmission media. Laser communication is of wide band. 
The laser diode is very widely used in fibre optics communication where it is used as optical carrier. The fibre optics system is featured with (1) improved transmission fidelity (2) increased data rate (3) increase in transmission distance between relay stations.

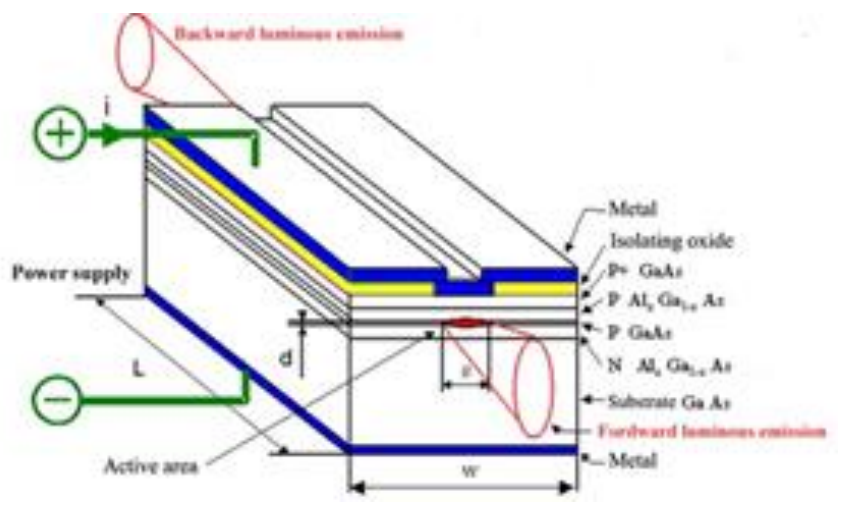

Figure 1. A GaAS laser

Fibre optic cables have military applications since it is highly secure. One cannot tap the cables without tampering or breaking the link. However security-wise line of sight laser beams are degrees ahead. The narrow laser beam allow high quality audio and is impossible for anybody to tap without knowledge of the designer. Undetectable by spectrum analyzers and RF meters, they can have very wide applications in communication and non-communication fields such as medical sciences, finance and in defence sector.

The pair of mirrors which reflect amplified light back and forth to form an optical cavity (called Fabry Perot resonator). It has a set of characteristics resonant frequencies. Under equilibrium, the optical power loss (which includes transmission loss at the mirrors) during one round trio through the active medium just balances the gain. Thus self-oscillation will start only after the gain exceeds the losses. This is what we call the lasing threshold.

This can be expressed, assuming steady-state operation [2] as

$$
\mathrm{R}_{1} \mathrm{R}_{2} \exp \left(2 \mathrm{~g}_{\text {threshold }}\right) \exp (-2 \alpha \mathrm{l})=1
$$

Here $R_{1}$ and $R_{2}$ are the mirror (power) reflectivity, $I$ is the length of the gain medium, $\exp \left(2 \mathrm{~g}_{\text {threshold }}\right)$ is the round-trip threshold power gain, and $\exp (-2 \mathrm{al})$ is the round trip power loss. Note that $\alpha>0$. This equation separates the losses in a laser into localised losses due to the mirrors, over which the experimenter has control, and distributed losses such as absorption and scattering. Optical losses being virtually constant near threshold, one can rewrite above equation as

$$
g_{\text {threshold }}=\alpha_{0}-1 /\left.2\right|^{*} \ln \left(R_{1} R_{2}\right)
$$

Since $R_{1} R_{2}>1$, both terms on the right side are positive, hence both terms increase the required threshold gain parameter. This means that minimising the gain parameter $g_{\text {threshold }}$ requires low distributed losses and high reflectivity mirrors. $\alpha_{0}$ generally increases with I due to diffraction losses.

Laser transmission in air, is inherently linked to laser spot size. Lasers usually emit beams with a Gaussian profile. A Gaussian beam is a beam of electromagnetic radiation whose transverse electric field and intensity (irradiance) distributions are described by Gaussian functions. Spot size is nothing but the radius of the beam itself. The irradiance of the beam decreases gradually at the edges. The distance across the centre of the beam for which the irradiance (intensity) equals $1 / \mathrm{e}^{2}$ of the maximum irradiance $\left(1 / \mathrm{e}^{2}=0.135\right)$ is defined as the beam diameter. The spot size $(w)$ of the beam is defined as the radial distance (radius) from the centre point of maximum irradiance to the $1 / \mathrm{e}^{2}$ point (see figure 2 next). 
Gaussian laser beams are said to be diffraction limited when their radial beam divergence is close to the minimum possible value, which is given by [3]

$$
\theta=\frac{\Theta}{2}=\frac{\lambda}{\pi w_{0}}
$$

Here $\lambda$ is the wavelength of the given laser and $w 0$ is the radius of the beam at the narrowest point, which is termed as the beam waist.

\section{Supplementary Facts From Literature}

Laser produced microstructures have influence over tribological behaviour of ceramics. [4]. Laser surface texturing is one of the most advanced field where tiny micro pores serve as store for the lubricants and can be used in case of deficit in lubricant conditions. They can be used for pressure bearing or traps for wear debris [5]. When pulsed laser beam passes through glass, it can create crater shaped pores in landing zone [6]. Laser intensities have become ultrahigh and have crossed $1000 \mathrm{~W} / \mathrm{cm}^{2}$ - a fourfold increase in 10 years [8].

Since laser requires smaller aperture compared to microwave radar, it can be commissioned in satellites for communication. However laser beam require special IR sensitive equipment, where alignment is of utmost importance. Potential bandwidth of radar using lasers can translate to very precision range measurement [9].

Lasers have been considered for space communications since their realization in 1960. Advancement in system architecture, data formatting and component technology have made laser communications in space a viable alternative to inter satellite link applications. Most important in space laser communications has been the development of a reliable, high power, single mode laser diode as a directly modulated laser source.

\section{Circuit Details and Working}

Figure 2 show us the transmitter as well as the receiver section. The transmitter circuit comprises condenser microphone transistor amplifier BC548 followed by an op-amp stage built around $\mu \mathrm{A} 741$.

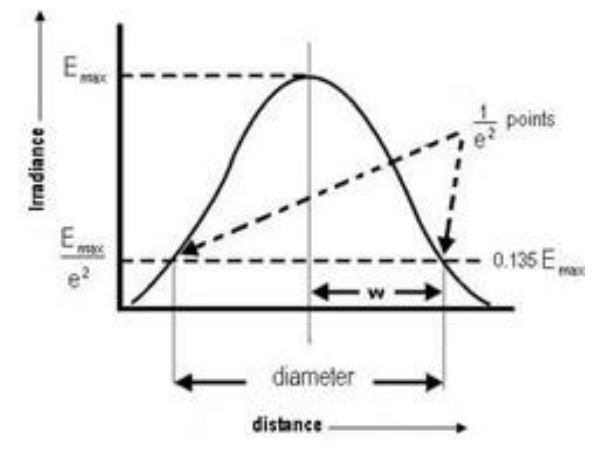

Figure 2. Transmitter as well as the receiver section

BC547 is a low cost general purpose NPN BJT, originated with metal cased BC108 family of transistors. The minimum performance characteristics expected are:

Breakdown voltage, collector to emitter with base open circuit (VCEO) $=45 \mathrm{~V}$

Collector Base voltage $(\mathrm{VCBO})=50 \mathrm{~V}$

Emitter Base voltage (VEBO) $=6 \mathrm{~V}$

Rated continuous collector current $\mathrm{IC}=100 \mathrm{~mA}$

Rated total power dissipation $=500 \mathrm{mV}$

Transition frequency (gain bandwidth product) $=150 \mathrm{MHz}$ minimum 
The current gain may be anywhere between $110 \& 800$

When we give a voice signal from the mike, it converts the voice signal into the electrical signal. This electrical signal is fed to IC741 (op-amp) for amplification. The gain of the op-amp can be controlled with the help of 1-mega-ohm potentiometer .

The AF output from IC is coupled to the base of a class B amplifier which, in turn, modulates the signal. Key design parameters for audio power amplifiers are frequency response, gain, noise and distortion. These are interdependent; increasing gain often leads to undesirable increases in noise and distortion. While negative feedback actually reduces the gain, it also reduces distortion. In high quality modern amplifiers the open loop response is at least $200 \mathrm{kHz}$ cancelling transient intermodulation distortion (TIM). Peter Baxandall's approach helped audio design engineers to better evaluate the distortion processes in amplifier.

BC557 is a PNP bipolar amplifier transistor, functionally identical to BC107-9, designed for use in audio switching and television circuits. Features include:

$V_{C E O}=-45 V, V_{C B O}=-50 V, V_{E B O}=-5 V, I_{C}=-100 \mathrm{~mA}$, Base Current (peak) $-200 \mathrm{~mA}$

The signal is amplified twice by using the combination of class B push pull amplifier. This electrical signal is fed to the laser where it is modulated into optical signal. This way beam is generated.

The transmitter uses 5V power regulated supply using IC 7805. However, the 3-volt laser torch (after removal of its battery) can be directly connected to the circuit-with the body of the torch connected to the class $\mathrm{B}$.

IC 7805 is a flexible, easy to implement, inexpensive device. It requires no additional components to provide a constant stable, regulated source of power, have protective shield against overheating and short circuiting.

The beam produced above travels through the free space medium and strikes the photodiode at the receiver section. The photodiode converts the optical signal into electrical signal and again this signal is amplified using IC741 and a combination of class B push pull amplifiers. The amplified signal is fed to the speaker which converts the electrical signal into voice signal and we get the output.

The receiver circuit uses an npn photodiode as the light sensor that is followed by a two-stage transistor preamplifier and IC741 based audio Power amplifier. The receiver does not need any complicated alignment. Just keep the photodiode oriented towards the remote transmitter's laser point and adjust the volume control for a clear sound. To avoid $50 \mathrm{~Hz}$ hum noise in the speaker, keep the photodiode away from AC light sources such as bulbs. The reflected sunlight, however, does not cause any problem. But the sensor should not directly face the sun. The diagrams of transmitter and receiver are shown consecutively in Figure 3 and 4.

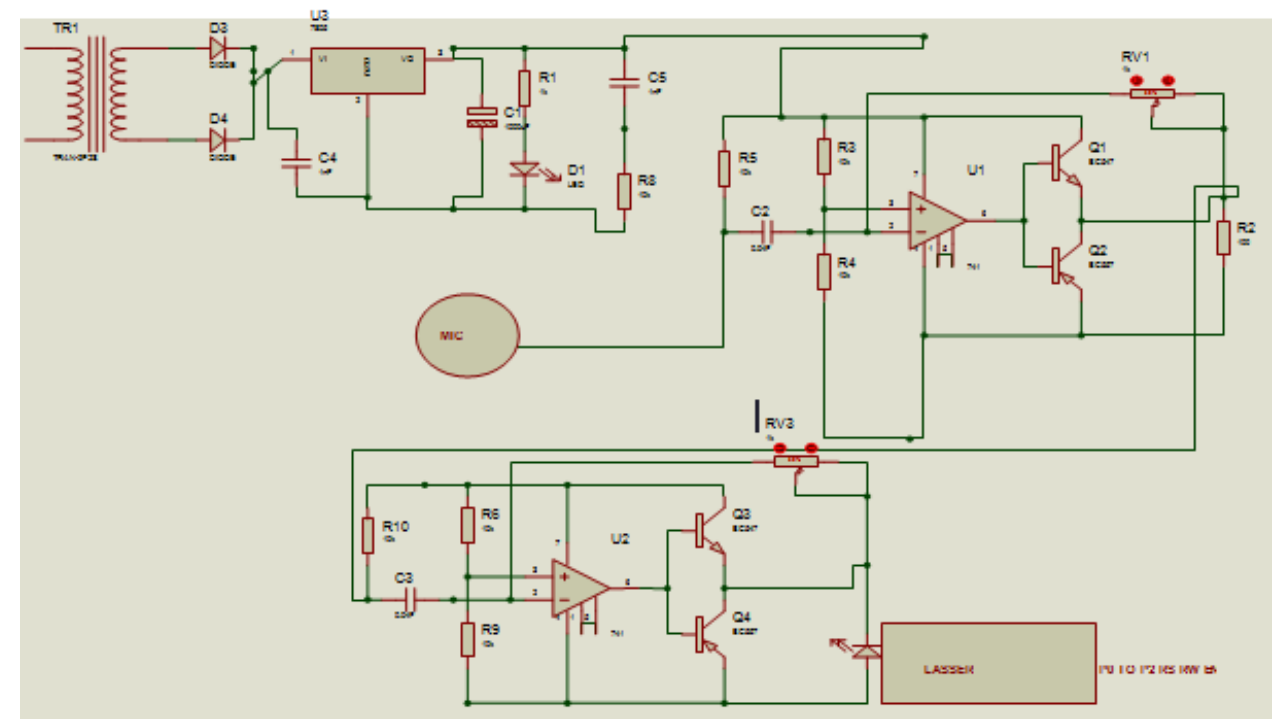

Figure 3. Diagrams of transmitter 


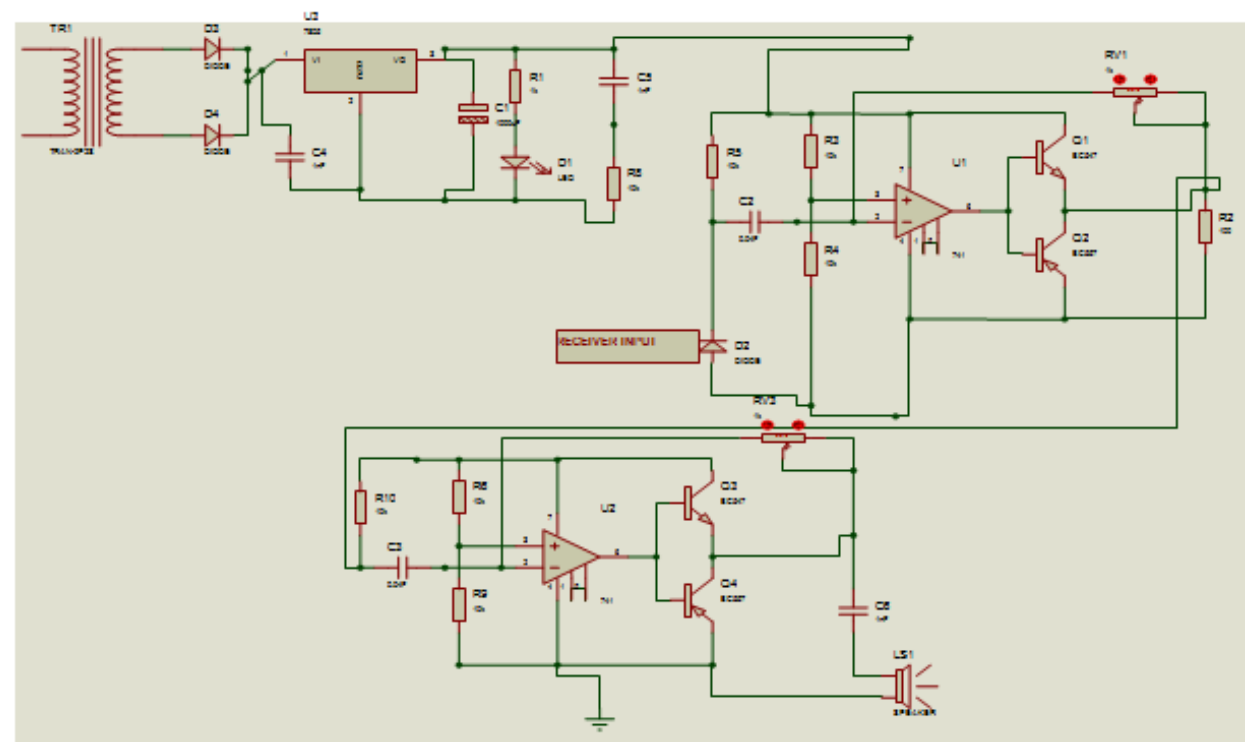

Figure 4. Diagrams of receiver

\section{Conclusion}

We had demonstrated line of sight communication exploring the laser features and properties. In future, laser technology will be applied in a big way in field of communication, more so because it does not requires any license. The project can be extended for a range for more than 500 meters with different powerful lasers, so used. This project can also be made by using optical fibre as the medium of transport which can be help in manipulating the path of ray in more cohesive manner.

\section{References}

[1] Robert L Byer Professor of applied physics and vice provost and dean of research, Stanford University, Stanford. Diode Laser-Pumped Solid-State Lasers (CA 94305 Science 12 Feb 1988; 239(4841): 742-747 DOI:10.1126/science.239.4841.74.2.

[2] Gerd Keiser: Optical Fiber Communica-tions, McGraw Hill, New Delhi, 2000

[3] J Wilson, JFB Hawkes: Optoelectronics, an Introduction, Prentice Hall of India Private Limited, New Delhi: 2003.

[4] M Geiger, S Roth, W Becker Influence of Laser Produced Microstructures on the Tribological Behaviour of Ceramics. Elsevier, Surface and Coating Technology. 1998; 101: 17-22.

[5] I Etsion. State of Art in Laser Surface Texturing. J. of Tribology Trans. ASME. 2005; 127: 248-253.

[6] R Ranjan, DN Lambeth, M Tromel, P Goglia, Y Li, Laser Texturing for Low Flying Height Media. J. of Applied Physics. 1991; 69: 5745-5747.

[7] SH Yun, C Boudoux, GJ Tearney, BE Bouma. High-speed wavelength-swept semiconductor laser with a polygon-scanner-based wavelength filter. Journal of the Optical Society of America. 2003; 28(20): 1981-1983. DOI10.1364/OL.28001981.

[8] A Braun, G Kern, X Liu, D Du, J Squier, G Mourow. Ültrahigh-intensity laser: physics of the extreme on a table top Springer, Berlin: 1994; 60: 248-265.

[9] K Govinda, Waseem Arshad, Santosh Ram, Shashank Agrawal. Laser Torch Based Voice Transmitter and Receiver. International Journal for Scientific Research and Development. 2014; 2(3): 397-399.

[10] Marc Rioux. Laser range finder based on synchronized scanners. Journal of the Optical Society of America. 1984; 23(21): 3837-3844. doi10.1364/AO.23.003837.

[11] Pere Colet, Rajarshi Roy. Digital communication with synchronized chaotic. Journal of the Optical Society of America. 1994; 19(24): 2056-2058. doi10.1364/OL19.002056.

[12] CV Shank Kaiser. Ultrashorf Laser Pulses and Applications. Springer-Verlag, Berlin. 1988; 60: 5-34,

[13] www.electronicsforyou.com

[14] http://www.scribd.com/doc/48917961/Laser-Torchbased-Voice-Transmitter-and-Receiver

[15] onlinepresent.org proceedings vol3 34 .pdf 